\title{
Correlation of Serum Uric Acid with Disease Activity and C-Reactive Protein in Patients Suffering from Rheumatoid Arthritis
}

\author{
Biswadip Ghosh', Debasish Baidya2 ${ }^{2}$, Pampa Halder ${ }^{3}$, Shamash Mandal4 \\ ${ }^{1}$ Department of Rheumatology, IPGME\&R and SSKM Hospital, Kolkata, India \\ ${ }^{2}$ Marwari Hospital and Research Centre, Guwahati, India \\ ${ }^{3}$ Department of Ophthalmology, RG Kar Medical College, Kolkata, India \\ ${ }^{4}$ Department of Medicine, BS Medical College, Bankura, India \\ Email: drbiswadip@gmail.com,drbaidyamdmed@gmail.com \\ drpampa07@gmail.com,drshyamash@gmail.com
}

Received 23 March 2016; accepted 28 August 2016; published 31 August 2016

Copyright (C) 2016 by authors and Scientific Research Publishing Inc.

This work is licensed under the Creative Commons Attribution International License (CC BY). http://creativecommons.org/licenses/by/4.0/

c) (i) Open Access

\section{Abstract}

Rheumatoid Arthritis is characterized by increased cardiovascular morbidity and mortality that cannot be fully explained by the presence of classical cardiovascular disease (CVD) risk factors suggesting that novel risk factors may play a role. Evidence accumulated over the past decade points that uric acid (UA) may be one such novel CVD risk factor in rheumatoid arthritis, and therefore one may hypothesize that UA may contribute to the increased CVD burden in RA. The current study attempted to correlate the levels of clinical disease activity index (CDAI), high sensitivity $\mathrm{C}$ reactive protein (hs-CRP) and uric acid in DMARD naive non-diabetic, non-hypertensive, euthyroid patients with normal lipid profile suffering from early and established Rheumatoid Arthritis before and after 3 months of DMARD therapy. It showed a positive and significant correlation among uric acid levels and CDAI and hs-CRP levels at diagnosis. There was significant reduction in the levels of CDAI, hs-CRP and UA after 3 months of DMARD therapy with significant correlation among the changes in the 3 parameters and the change in Uric Acid also correlated with the levels of CDAI and hs-CRP at diagnosis. We propose to measure uric acid both as acute phase reactant and marker for cardiovascular morbidity in patients suffering from Rheumatoid Arthritis.

\section{Keywords}

Uric Acid, Rheumatoid Arthritis, CDAI, CRP

How to cite this paper: Ghosh, B., Baidya, D., Halder, P. and Mandal, S. (2016) Correlation of Serum Uric Acid with Disease Activity and C-Reactive Protein in Patients Suffering from Rheumatoid Arthritis. Open Journal of Rheumatology and Autoimmune Diseases, 6, 79-84. http://dx.doi.org/10.4236/ojra.2016.63013 


\section{Introduction}

Rheumatoid Arthritis (RA) is a systemic disease, affects approximately $0.5 \%$ - $1 \%$ of the adult population worldwide and may result in a variety of extra articular manifestations, including fatigue, subcutaneous nodules, pleuropulmonary involvement, cardiovascular disease, peripheral neuropathy, vasculitis, and hematologic abnormalities [1].

Morbidity and mortality rates are higher in individuals with rheumatoid arthritis than in the general population. Cardiovascular diseases now represent one of the most common causes of death in RA. RA appears to represent an independent risk factor for ischemic heart disease, like diabetes mellitus [1].

Recent studies have shown increased level of C-reactive protein in patients with cardiovascular disease. Patients with RA have high C-reactive protein level which correlates with disease activity and as mentioned earlier, there is increased incidence of cardiovascular disease in patients of RA. It has also been shown that increased serum uric acid level is associated with increased chances of cardiovascular disease. However, the increased cardiovascular morbidity and mortality in rheumatoid arthritis cannot be fully explained by classical cardiovascular risk factors, suggesting that other novel risk factors may play a role. Apart from the various mediators of inflammation like interleukins, uric acid is being thought to be, and, investigated as one such novel mediator and risk factor for cardiovascular diseases in RA.

If uric acid has a role in the pathogenesis of cardiovascular disease in RA, then, with change in disease activity, uric acid level is likely to change and correlate with the change in disease activity and the inflammatory milieu.

The primary aims of the study are to assess the disease activity, high sensitivity C-reactive protein level and serum uric acid level at diagnosis, reassess the 3 parameters after 3 months of Disease Modifying Anti-Rheumatic Drugs (DMARD) therapy in DMARD naive rheumatoid arthritis patients and correlate the changes in the 3 parameters if any.

\section{Materials and Method}

120 newly diagnosed DMARD naive patients suffering from rheumatoid arthritis were taken in the study, done at Rheumatology OPD of Medical College, Kolkata. Patients having diabetes, Hypertension, dyslipidemia, liver, kidney, thyroid dysfunction, any documented previous or current cardiac illness or family history of coronary artery disease or hyperuricemia, pregnancy, or with coexisting disease associated with high uric acid levels e.g., malignancy, chronic renal failure, active chronic infections e.g. Tuberculosis were excluded. Known smoker or alcoholics were also excluded.

Patients were diagnosed of having RA using ACR 2010 criteria. CDAI [2] (clinical disease activity index = swollen joint count +tender joint count +patient's global activity + evaluator's global activity) was calculated. Besides routine investigations, serum uric acid (by uricase method) and hs-CRP (by nephelometry) were also measured, at diagnosis and again after three months.

The study got clearance from Institutional Ethics Committee.

Informed consent was taken from all the patients.

Patients were started on combination DMARDS from the onset in order to achieve rapid control of disease activity. The following DMARDs were used: Methotrexate, Sulfasalazine, and Hydroxychloroquine.

All patients received Methotrexate and Sulfasalazine. Hydroxychloroquine was added to the combination in patients with CDAI $>22$ at diagnosis.

Methotrexate was given in a dose of 7.5 - $15 \mathrm{mg}$ once weekly with folic acid supplementation of $5 \mathrm{mg}$ once weekly with a gap of 1 day between the methotrexate dose and dose of folic acid.

Sulfasalazine was started in a dose of 500mg twice daily with dose escalation of $500 \mathrm{mg}$ weekly to a maximum of 2 g/day.

Hydroxychloroquine was given in a dose of 200 - $400 \mathrm{mg}$ with maximum daily dose not exceeding $6.5 \mathrm{mg} / \mathrm{kg}$ body weight.

No patients were given steroids as it may influence the parameters.

NSAIDs were given for the initial 2 - 4 weeks of treatment for symptom control. Proton-pump inhibitors were also given throughout the duration of treatment. Patients were advised to start physical therapy when feasible. Patients were followed up at monthly intervals. 
Disease activity was assessed at presentation and after 3 months of DMARD therapy using CDAI [2]. High disease activity was when CDAI $>22$, moderate $>10$ but $<22$, low $<10$.

The level of uric acid was assessed in relation to CDAI and the level of hs-CRP, at presentation and at the end of 3 months of DMARD therapy. Any change in uric acid level was correlated with respective CDAI and hsCRP value.

Early RA was defined as patients with disease duration less than 6 months while those with disease duration more than 6 months were labeled as having Established RA. The normal level of hs-CRP was taken as a value less than $3 \mathrm{mg} / \mathrm{L}$ and the normal values of Uric Acid taken as $2.4-6 \mathrm{mg} / \mathrm{dl}$ in females and 3.4 - 7/dl in males.

Results were tabulated in Microsoft Office Excel worksheet and expressed as mean \pm standard deviation for continuously distributed variable, and in absolute numbers and percentages for discrete variables. $p<0.05$ was considered statistically significant. Graphpad online software was used for statistical analysis.

\section{Results and Analysis}

Total number of participants in the study was 120 . Female patients constituted $70 \%(\mathrm{n}=84)$ of the study population. Mean age of the study group was $40.33 \pm 12.05$ yrs. 16 males and 34 females had early RA whereas 20 males and 50 females had established RA.

Patients with known cardiovascular disease, hypertension, diabetes and dyslipidemia were excluded from the study. Known hypothyroid and patients with renal/hepatic dysfunction were also excluded. All the patients were DMARD naive at the time of diagnosis and initiation of therapy. None of the patients reported any serious adverse events during the study and none withdrew from the study or discontinued therapy during the study.

Mean CDAI at diagnosis is $39.27 \pm 12.46$, with higher values in early RA and males showing higher values in overall and early RA. Mean hs-CRP level at diagnosis is $7.905 \pm 6.084 \mathrm{mg} / \mathrm{L}$ with higher values in early RA and in females. Mean uric acid level at diagnosis to be $4.738 \pm 1.395 \mathrm{mg} / \mathrm{dl}$ with higher value in early disease and in females (Table 1).

Table 2 shows that there has been decrease in mean CDAI, hs-CRP and Uric Acid in all categories after 3 months of DMARD therapy.

Table 3 shows that with higher age there is higher disease activity, hs-CRP and Uric Acid level at diagnosis. Also there are greater changes in the parameters after 3 month of DMARD therapy in the older age groups.

Table 4 shows that there has been significant decrease in CDAI, hs-CRP and Uric Acid from the values at diagnosis after 3 months of DMARD therapy.

Table 1. Mean values of CDAI, hs-CRP (mg/L) and Uric Acid (mg/dl) at diagnosis.

\begin{tabular}{|c|c|c|c|c|c|c|c|c|}
\hline \multicolumn{3}{|c|}{ CDAI at Diagnosis } & \multicolumn{3}{|c|}{ hs-CRP at Diagnosis } & \multicolumn{3}{|c|}{ Uric Acid at Diagnosis } \\
\hline Male & Female & $\begin{array}{l}\text { Male + } \\
\text { Female }\end{array}$ & Male & Female & $\begin{array}{l}\text { Male + } \\
\text { Female }\end{array}$ & Male & Female & $\begin{array}{c}\text { Male + } \\
\text { Female }\end{array}$ \\
\hline
\end{tabular}

Early RA $43.13 \pm 13.0141 .65 \pm 12.6642 .12 \pm 12.528 .475 \pm 2.2879 .776 \pm 10.4769 .360 \pm 8.6644 .975 \pm 0.5805 .112 \pm 1.507 \quad 5.068 \pm 1.272$

Established RA

$37.00 \pm 9.2737 .32 \pm 13.3437 .23 \pm 12.196 .590 \pm 2.3746 .976 \pm 3.1616 .866 \pm 2.9284 .500 \pm 1.1844 .504 \pm 1.5644 .503 \pm 1.1449$

Early+

Established $39.72 \pm 11.1839 .07 \pm 13.0939 .27 \pm 12.46 \quad 7.428 \pm 2.463 \quad 8.110 \pm 7.1147 .905 \pm 6.0844 .711 \pm 0.9694 .750 \pm 1.553 \quad 4.738 \pm 1.395$ RA

Table 2. Mean values of CDAI, hs-CRP (mg/L) and Uric Acid (mg/dl) after 3 months of treatment (DMARD therapy).

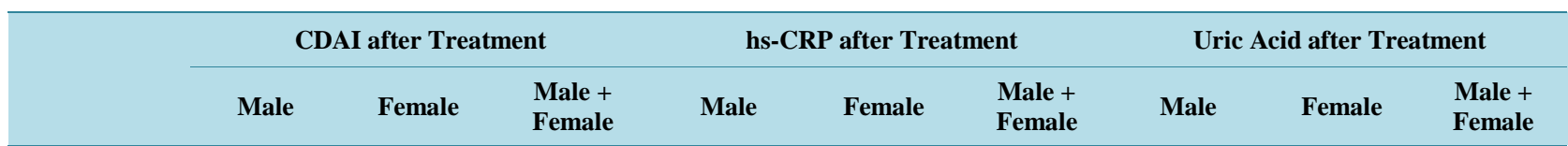

Early RA $\quad 27.00 \pm 11.4025 .18 \pm 10.8025 .76 \pm 10.794 .863 \pm 1.4576 .441 \pm 7.8815 .936 \pm 6.5263 .113 \pm 0.7223 .288 \pm 0.9433 .232 \pm 0.867$

Established RA $20.00 \pm 7.42 \quad 20.92 \pm 9.50 \quad 20.66 \pm 8.85 \quad 4.430 \pm 1.566 \quad 4.11 \pm 1.933 \quad 4.206 \pm 1.8193 .170 \pm 1.0352 .980 \pm 0.9823 .034 \pm 0.986$

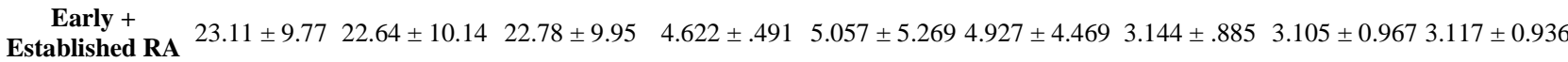


Table 3. Age-wise distribution of the values of CDAI, hs-CRP (mg/L) and Uric Acid (mg/dl) at diagnosis and their corresponding changes after 3 months of treatment.

\begin{tabular}{ccccccc}
\hline \multirow{2}{*}{$\begin{array}{c}\text { AGE in } \\
\text { Year }\end{array}$} & At Diagnosis & $\begin{array}{c}\text { Change after } \\
\text { Treatment }\end{array}$ & At Diagnosis & $\begin{array}{c}\text { Change after } \\
\text { Treatment }\end{array}$ & At Diagnosis & $\begin{array}{c}\text { Hs-CRP (mg/L) } \\
\text { Treatment }\end{array}$ \\
\cline { 2 - 7 } & $\mathbf{2}$ & $14.50 \pm 6.41$ & $6.900 \pm 2.194$ & $1.800 \pm 1.343$ & $4.017 \pm 0.387$ & $1.283 \pm 0.483$ \\
$\mathbf{2 5}$ & $34.00 \pm 15.44$ & $16.29 \pm 4.90$ & $7.036 \pm 2.487$ & $2.943 \pm 1.381$ & $4.579 \pm 1.349$ & $1.486 \pm 0.715$ \\
$\mathbf{2 5} \mathbf{- 4 0}$ & $38.93 \pm 12.27$ & $16.77 \pm 5.33$ & $9.073 \pm 8.787$ & $3.277 \pm 2.722$ & $5.077 \pm 1.525$ & $1.885 \pm 1.088$ \\
$>\mathbf{4 0}$ & $40.85 \pm 12.11$ & & & & &
\end{tabular}

Table 4. Correlation of CDAI, hs-CRP and Uric Acid at diagnosis with the respective changes in the parameters after 3 months of DMARD therapy.

\begin{tabular}{cccc}
\hline & $\begin{array}{c}\text { CDAI at Diagnosis } \rightarrow \text { Change } \\
\text { in CDAI }\end{array}$ & $\begin{array}{c}\text { hs-CRP at Diagnosis } \rightarrow \text { Change } \\
\text { in hs-CRP }\end{array}$ & $\begin{array}{c}\text { Uric Acid at Diagnosis } \rightarrow \text { Change } \\
\text { in Uric Acid }\end{array}$ \\
\hline Correlation Coefficient & 0.6272 & 0.8423 & 0.7504 \\
P Value & $<0.0001$ & $<0.0001$ & $<0.0001$ \\
\hline
\end{tabular}

\section{Discussion}

Our study was prospective in nature.

We use simple tool like CDAI to measure disease activity in patients suffering from RA. We are trying to find out other simple and useful means to assess disease activity and at the same time, to care for their comorbidities and disability limitation.

A cross-sectional study by Panoulas V. F. et al. suggests that SUA may be independently associated with CVD in RA patients [3]. A study done on a large cohort of unselected outpatients in Italy has shown that hyperuricaemia is associated with increased C-reactive protein concentrations [4]. It is also reported in literature that elevated serum urate in the absence of gout was strongly associated with markers of systemic inflammation [5].

In our study we looked for the parameters at both Early RA and Established RA.

CDAI is an established tool to assess disease activity and already validated in Indian patients suffering from RA [2].

The mean CDAI at diagnosis was $39.27 \pm 12.46$, most of the subjects showing high disease activity and higher values in early RA. The mean hs-CRP level at diagnosis was $7.905 \pm 6.084 \mathrm{mg} / \mathrm{L}$ with higher values in females and early RA. All the subjects had high hs-CRP values at diagnosis, which probably indicates a higher inflammatory milieu early in the disease. The mean uric acid level at diagnosis was $4.738 \pm 1.395 \mathrm{mg} / \mathrm{dl}$ with higher value in females and in early disease.

Patients with higher age (>40 years) had higher disease activity, hs-CRP and Uric Acid levels at diagnosis and showed greater changes in the parameters after 3 months of DMARD therapy.

Uric Acid showed a positive correlation with both CDAI and hs-CRP at diagnosis with extremely high statistical significance. In the sub group analysis, CDAI correlated with Uric Acid significantly in females and established RA while Uric Acid correlated with hs-CRP significantly in both sexes and in both early and established RA.

At diagnosis, there was statistically significant positive correlation between uric acid and CDAI > 22 while the correlation in case of CDAI $<22$ was not statistically significant. This may be due to the fact that only 8 subjects had CDAI less than 22 at diagnosis and a statistical analysis on such a small number of subjects is not likely to be actual representation of the population as a whole. This is also the likely explanation in case of the statistically non-significant correlation between changes in Uric Acid after 3 months of DMARD therapy with CDAI $<22$ at diagnosis.

There was significant change in CDAI, hs-CRP and Uric Acid levels after 3 months of DMARD therapy and it was higher in early RA. Females showed a higher change in hs-CRP and Uric Acid after 3 months of DMARD therapy while males had a greater change in CDAI. There was statistically significant positive correlation between the changes in Uric Acid after 3 months of DMARD therapy and the levels of CDAI and hs-CRP at diag- 
nosis. Also there was statistically significant correlation between the changes in CDAI, hs-CRP and Uric Acid.

Males showed statistically significant correlation between change in Uric acid and change in hs-CRP after 3 months of DMARD therapy and also with the hs-CRP levels at diagnosis. Females showed statistically significant correlation between change in Uric acid and change in both CDAI and hs-CRP after 3 months of DMARD therapy and also with the CDAI and hs-CRP levels at diagnosis. This might either be due to the small number of male subjects in the study or indicate that male sex may have different influence over the Uric Acid dynamics.

However, there was no statistically significant correlation between the levels of Uric Acid, CDAI or hs-CRP reached after 3 months of DMARD therapy including the sub-groups, probably reflecting the fact that not all patients respond to similar extent to all the DMARDs used in the treatment and different DMARD probably has different extent of influence on the inflammatory milieu and anti-atherogenic effect and thus the heterogeneity in the changes in uric acid levels leading to statistically insignificant results.

According to our knowledge, the role of uric acid in the pathogenesis of cardiovascular disease in RA requires in-depth research. The possible role of uric acid in the pathogenesis of CVD in RA was beyond the scope of the present study, as the aim was to identify which of the parameters easily obtained in daily routine is an additional risk factor for cardiac involvement in RA.

We propose to measure uric acid both as acute phase reactant and marker for cardiovascular morbidity, which may be interfered upon by acting on disease activity and urate lowering therapy.

Future studies are necessary before we can state for certain whether uric acid is simply a biomarker or whether it has a true pathophysiological role in systemic inflammation. If the latter is true, uric acid may emerge as one of the major cardiovascular risk factors [6]. Worse, it is also being discussed that raised hs-CRP and Uric acid are associated with other components of metabolic syndrome like lipid, glucose or obesity [7].

During selection of patients, probable bias e.g. choosing patients from a single department or OPD was avoided, because all patients suspected or diagnosed as RA were referred to our Rheumatology Clinic from all the departments in the hospital during our study period.

Intra or inter observer bias for the laboratory values has been avoided by sending the patients to the hospital laboratory only. All probabilities of inter-observer bias or intra-observer bias during various investigations and examinations were ignored.

There were no missing data and every participant underwent clinical examination and investigations on the same day.

\section{Limitations}

The study has been conducted on a small group of patients.

No control group was included in the study.

Only hs-CRP taken as representative of the inflammatory status was used whereas there were a number of inflammatory markers related to disease activity in RA patients.

The influence of individual DMARD was not assessed in the study.

Since the established cardiovascular risk factors were excluded instead of including them, their additive effect on uric acid level could not be assessed.

\section{References}

[1] Kaplan, M.J. (2010) Complications of Rheumatoid Arthritis-Assessment, Prevention and Treatment. Rheumatic Disease Clinics, 36, 405-426. http://dx.doi.org/10.1016/j.rdc.2010.02.002

[2] Ghosh, A., Ghosh, A., Pain, S., Pande, A., et al. (2011) Comparison between DAS28, CDAI and HAQ-DI as Tools to Monitor Early Rheumatoid Arthritis Patients in Eastern India. Indian Journal of Rheumatology, 6, 116-122.

[3] Panoulas, V.F., Douglas, K.M., Milionis, H.J., Nightingale, P., Kita, M.D., Klocke, R., Metsios, G.S., Stavropoulos-Kalinoglou, A., Elisaf, M.S. and Kitas, G.D. (2007) Association of Serum Uric Acid with Cardiovascular Disease in Rheumatoid Arthritis. Rheumatology (Oxford), 46, 1466-1470. http://dx.doi.org/10.1093/rheumatology/kem159

[4] Lippi, G., Targher, G., Montagnana, M., Salvagno, G.L., et al. (2008) Hyperuricaemia Is Associated with Increased C-Reactive Protein Concentrations in a Large Cohort of Unselected Outpatients. Nutrition, Metabolism \& Cardiovascular Diseases, 18, e41-e42. http://dx.doi.org/10.1016/j.numecd.2008.03.011

[5] Pineda, C., Amezcua-Guerra, L.M., Solano, C., et al. (2011) Joint and Tendon Subclinical Involvement Suggestive of Gouty Arthritis in Asymptomatic hypeRuricemia: An Ultrasound Controlled Study. Arthritis Research \& Therapy, 13, 
R4. http://dx.doi.org/10.1186/ar3223

[6] Inaba, S., Sautin, Y., Garcia, G.E. and Johnson, R.J. (2013) What Can Asymptomatic Hyperuricaemia and Systemic Inflammation in the Absence of Gout Tell Us? Rheumatology, 52, 963-965. http://dx.doi.org/10.1093/rheumatology/ket001

[7] Sah, S.K., Khatiwada, S., Pandey, S., Kc, R., et al. (2016) Association of High-Sensitivity C-Reactive Protein and Uric Acid with the Metabolic Syndrome Components. SpringerPlus, 5, 269. http://dx.doi.org/10.1186/s40064-016-1933-y

\section{Submit or recommend next manuscript to SCIRP and we will provide best service for you:}

Accepting pre-submission inquiries through Email, Facebook, LinkedIn, Twitter, etc.

A wide selection of journals (inclusive of 9 subjects, more than 200 journals)

Providing 24-hour high-quality service

User-friendly online submission system

Fair and swift peer-review system

Efficient typesetting and proofreading procedure

Display of the result of downloads and visits, as well as the number of cited articles

Maximum dissemination of your research work

Submit your manuscript at: http://papersubmission.scirp.org/ 